\title{
Absolute point algorithm for solving unbalanced fuzzy transportation problem
}

\section{K. Rathi' ${ }^{1}$, S. Muruganantham ${ }^{2}$}

${ }^{1}$ Associate Professor, Department of Mathematics, Velalar College of Engineering and Technology, Erode, Tamil Nadu, India, 638012.

${ }^{2}$ Assistant Professor and Head, Department of Computer Technology and Information Technology, Kongu Arts and Science College, Erode, Tamil Nadu, India, 638107.

\section{ABSTRACT}

In real time situations, the total availability of goods or product may be more or less than the actual market demand and the unbalanced transportation situation arise more commonly. Such unbalanced Transportation Problems (TP) are solved by introducing dummy source or destination which do not exist in reality. The optimal allocation involves cells from such dummy source or destination and the allocated number of quantities are held back at one or more origins. The paper aims to propose an algorithm based on Absolute Points to solve unbalanced TP under fuzzy environment. The proposed algorithm is advantageous than the existing algorithms in such a way that it provides the added information of transporting the excess availability from dummy supply point to appropriate destination to meet future demands at minimum cost. Finally, by virtue of the proposed algorithm an example is done to illustrate the practicality and the effectiveness of the proposed algorithm.

Keywords:Transportation management; fuzzy transportation problem; absolute point; heptagonal fuzzy numbers

\section{Introduction}

In current scenario due to the globalisation of world market, transportation of goods from one place to another becomes increasingly important. It is mandatory for any company to have a sound knowledge about the transportation system for the efficient movement of raw materials and finished goods. Therefore the present work is focused to look for the transportation system that aims in reducing cost and improving service.

The Transportation problem was first formulated by Hitchcock (1941). Later many researchers worked on this topic and developed variants in basic Transportation problem.In general, transportation problems are solved with the assumptions that the parameters of the transportation problem such as unit cost of transportation from each source to each destination, availability of the product at each source and demand at each destination are specified in a precise way and the problem is dealt in crisp environment. But in reality, the parameters of the transportation problem are not always exactly known and stable. This imprecision may follow from the lack of exact information, uncertainty in judgment etc. Therefore, Zadeh (1965) introduced the concept of fuzzy numbers.

Saad and Abbas (2003) developed an algorithm to solve the transportation problems in fuzzy environment. Vogel's approximation method was fuzzified by Das \& Baruah (2007) and found the fuzzy initial basic feasible solution of fuzzy transportation problems in which all the parameters are represented by triangular fuzzy numbers. Basirzadeh (2011) used the classical algorithms to find the fuzzy optimal solution of fully fuzzy transportation problems by transforming the fuzzy parameters into crisp parameters. Using simplex- type algorithm proposed by Arsham \& Khan (1989), Gani et al (2011) obtained the fuzzy optimal solution of fuzzy transportation problems having parameters as trapezoidal fuzzy numbers.

While solving unbalanced transportation problems two cases may be observed. The total availability is more than the total demand or vice-versa. In case of excess availability, sometimes it may happen that we do not have enough storage place for the excess commodityat the source(s) and wish to transport it to the destination(s) for the future 
demand (Deepika Rani et al. 2014). To solve such problems a dummy destination is added, where the excess availability is transported. Since the dummy destination does not have any existence in reality, it is not possible to find, to which destination the excess available product should be transported at a minimum cost.

In this paper, an algorithm based on Absolute Points (AP) is proposed to solve unbalanced Transportation problem under fuzzy environment. The fuzziness in the transportation parameters supply and demand are represented by Heptagonal fuzzy numbers (Rathi \& Balamohan, 2014). The proposed algorithm is advantageous than the existing algorithms in such a way that it is suitable for both balanced as well as unbalanced FTP without the necessity of introducing dummy supply or destination. An illustration is given to show the effectiveness of the proposed algorithm.

\section{Preliminaries}

In this section, the definitions of fuzzy numbers including symmetric heptagonal fuzzy numbers, its arithmetic operations and ranking function are given (Rathi \& Balamohan, 2016).

\section{Definition 2.1 Fuzzy Number}

A fuzzy set $\widetilde{\mathrm{A}}$ is a fuzzy number iff (i) For all $\alpha \in(0,1]$ the $\alpha$-cutsets $\mathrm{A}_{\alpha}$ is a convex set(ii) $\mu_{\widetilde{\mathrm{A}}}$ is an upper semi continuous function(iii) $\operatorname{supp}(\widetilde{A})$ is a bounded set in $R$ (iv) The height of $\widetilde{A}=\max _{x \in X} \mu_{\widetilde{A}}(x)=\omega>0$

\section{Definition 2.2 Symmetric Heptagonal Fuzzy Numbers}

The symmetric HFN is defined as $\tilde{\mathrm{H}}_{\mathrm{sym}}=(\tilde{\mathrm{h}} ; \mathrm{k}, \omega)_{\beta}$ where the ordinates are given by $\mathrm{h}_{\mathrm{i}}=\mathrm{h}+(\mathrm{i}-4) \beta$ for $\mathrm{i}=1$ to 7 and $\beta$ denotes the equal space between the ordinates. The membership function of $\tilde{\mathrm{H}}_{\text {sym }}$ is given as follows

$$
\mu_{\tilde{H}_{\text {sym }}}(x)=\left\{\begin{array}{cl}
0 & \text { for } x<h-3 \beta \\
k\left(\frac{x-(h-3 \beta)}{\beta}\right) & \text { for } h-3 \beta \leq x \leq h-2 \beta \\
k & \text { for } h-2 \beta \leq x \leq h-\beta \\
k+(\omega-k)\left(\frac{x-(h-\beta)}{\beta}\right) & \text { for } h-\beta \leq x \leq h \quad \text { where } 0<k<1, k \leq \omega \leq 1 \\
k+(\omega-k)\left(\frac{(h+\beta)-x}{\beta}\right) & \text { for } h \leq x \leq h+\beta \\
k\left(\frac{(h+3 \beta)-x}{\beta}\right) & \text { for } h+\beta \leq x \leq h+2 \beta \\
0 & \text { for } h+2 \beta \leq x \leq h+3 \beta \\
& \text { for } x \geq h+3 \beta
\end{array}\right.
$$

\section{Definition 2.3 Arithmetic Operations on Symmetric HFN}

Let $\widetilde{\mathrm{A}}=\left(\widetilde{\mathrm{a}} ; \mathrm{k}_{\mathrm{A}}, \omega_{\mathrm{A}}\right)_{\beta_{1}}$ and $\widetilde{\mathrm{B}}=\left(\widetilde{\mathrm{b}} ; \mathrm{k}_{\mathrm{B}}, \omega_{\mathrm{B}}\right)_{\beta_{2}}$ be the two SymHFNs. The arithmetic operations on $\tilde{\mathrm{A}}$ and $\tilde{\mathrm{B}}$ is defined as follows:

(i) Addition of two SymHFNs:

$$
\widetilde{\mathrm{A}}_{\mathrm{sym}} \oplus \widetilde{\mathrm{B}}_{\mathrm{sym}}=\left(\widetilde{\mathrm{a}+\mathrm{b}} ; \min \left\{\mathrm{k}_{\mathrm{A}}, \mathrm{k}_{\mathrm{B}}\right\}, \min \left\{\omega_{\mathrm{A}}, \omega_{\mathrm{B}}\right\}\right)_{\beta_{1}+\beta_{2}}
$$

(ii) Subtraction of two SymHFNs:

$$
\widetilde{\mathrm{A}}_{\text {sym }} \ominus \widetilde{\mathrm{B}}_{\mathrm{sym}}=\left(\widetilde{\mathrm{a}-\mathrm{b}} ; \min \left\{\mathrm{k}_{\mathrm{A}}, \mathrm{k}_{\mathrm{B}}\right\}, \min \left\{\omega_{\mathrm{A}}, \omega_{\mathrm{B}}\right\}\right)_{\beta_{1}+\beta_{2}}
$$

(iii)Scalar Multiplication of two SymHFNs:

$$
\lambda \widetilde{\mathrm{A}}_{\mathrm{sym}}=\left(\widetilde{\lambda \mathrm{a}} ; \mathrm{k}_{\mathrm{A}}, \omega_{\mathrm{A}}\right)_{\lambda \beta_{1}}
$$

(iv)Multiplication of two SymHFNs:

$\widetilde{\mathrm{A}}_{\text {sym }} \otimes \widetilde{\mathrm{B}}_{\text {sym }}=\left(\widetilde{\mathrm{ab}} ; \min \left\{\mathrm{k}_{\mathrm{A}}, \mathrm{k}_{\mathrm{B}}\right\}, \min \left\{\omega_{\mathrm{A}}, \omega_{\mathrm{B}}\right\}\right)_{\mathrm{b} \beta_{1}+\mathrm{a} \beta_{2}+(\mathrm{i}-4) \beta_{1} \beta_{2}}$ where $\mathrm{j}=1$ to 7

\section{Definition 2.4 Centroid formulae for the Symmetric HFN}

Let $\tilde{\mathrm{H}}_{\mathrm{sym}}=(\tilde{\mathrm{h}} ; \mathrm{k}, \omega)_{\beta}$ be a symmetric HFN where $\beta$ denotes the equal space between the ordinates and the 
ordinates are given by $\mathrm{h}_{\mathrm{i}}=\mathrm{h}+(\mathrm{i}-4) \beta$ for $\mathrm{i}=1$ to 7 . The pair of centroid formulae $\left(\overline{\mathrm{x}_{0}}, \overline{\mathrm{y}_{0}}\right)$ for the symmetric HFN $\tilde{\mathrm{H}}$ is $\overline{\mathrm{x}_{0}}(\tilde{\mathrm{H}})=\mathrm{h}$ and $\overline{\mathrm{y}_{0}}(\widetilde{\mathrm{H}})=\frac{1}{3}\left[\frac{5 \mathrm{k}^{2}+\omega(\omega+\mathrm{k})}{\omega+4 \mathrm{k}}\right]$.

Definition 2.5 Ranking method

Let $\tilde{\mathrm{H}}_{\text {sym }}$ be a symmetric HFN and let $\left(\overline{\mathrm{x}_{0}}, \overline{\mathrm{y}_{0}}\right)$ be the centroid of $\tilde{\mathrm{H}}_{\mathrm{sym}}$. Then the ranking function of $\tilde{\mathrm{H}}_{\text {sym }}$ which maps the set of all fuzzy numbers to a set of real numbers is defined as $\mathfrak{R}(\tilde{\mathrm{H}})=\overline{\mathrm{x}}_{0} \cdot \overline{\mathrm{y}}_{0} \quad$ which is the area between the centroid and the origin. The ranking method for HFN involves the following rules:

(i) $\tilde{\mathrm{A}} \prec \tilde{\mathrm{B}}$ if and only if $\mathfrak{R}(\tilde{\mathrm{A}})<\mathfrak{R}(\tilde{\mathrm{B}})$, (ii) $\tilde{\mathrm{A}} \succ \tilde{\mathrm{B}}$ if and only if $\mathfrak{R}(\tilde{\mathrm{A}})>\mathfrak{R}(\tilde{\mathrm{B}})$,

(iii) $\widetilde{\mathrm{A}}=\widetilde{\mathrm{B}}$ if and only if $\mathfrak{R}(\widetilde{\mathrm{A}})=\mathfrak{R}(\widetilde{\mathrm{B}})$.

\section{Mathematical formulation of fuzzy transportation problem}

The Fuzzy Transportation problem (FTP) can be mathematically stated as follows

Minimize

$$
\tilde{\mathrm{Z}}=\sum_{\mathrm{i}=1}^{\mathrm{n}} \sum_{\mathrm{j}=1}^{\mathrm{n}} \mathrm{C}_{\mathrm{ij}} \tilde{\mathrm{x}}_{\mathrm{ij}}
$$

subject to

$$
\sum_{\mathrm{j}=1}^{\mathrm{n}} \tilde{\mathrm{x}}_{\mathrm{ij}} \approx \tilde{\mathrm{a}}_{\mathrm{i}}, \mathrm{i}=1,2 \ldots \mathrm{m}
$$

$$
\sum_{\mathrm{i}=1}^{\mathrm{n}} \tilde{\mathrm{x}}_{\mathrm{ij}} \approx \tilde{\mathrm{b}}_{\mathrm{j}}, \mathrm{j}=1,2 \ldots \mathrm{n}
$$

where

- the cost $C_{\mathrm{ij}}$ is the cost of transporting one unit of a product from ith source to the $\mathrm{jth}$ destination and the information about the cost parameter is assumed to be certain.

- $\tilde{a}_{i}$ denotes the uncertain number of units available at ith source and the uncertainty is represented by Symmetric Heptagonal fuzzy number (symHFN).

- $\quad \tilde{b}_{\mathrm{j}}$ denotes the uncertain number of units required at jth destination and the uncertainty is represented by symHFN.

- the decision variable $\tilde{\mathrm{x}}_{\mathrm{ij}}$ denotes the uncertain number of units to be transported from ith source to the $\mathrm{jth}$ destination.

\section{Absolute points}

\section{Definition (Adlakha and Kowalski 1998)}

An absolute point (AP) is a location (q,r) in a Transportation matrix that must be loaded in any optimal solution regardless of the values $\tilde{a}_{i}$ and $\tilde{b}_{j}$. If such location $(q, r)$ is excluded in the solution then there would be a path leading to the location (q,r) from every other location that is loaded.

\subsection{Absolute point procedure}

The procedure for finding the absolute points is presented from (Adlakha and Kowalski 1998)

Step I: Form the matrix $d_{i j}^{k}=C_{k j}-C_{i j}$, for $i \neq k ; i=1,2, \cdots, m ; j=1,2, \cdots, n$.

Step II: Find the minimum element $e_{i}^{k}$ in each row of every matrix $d_{i j}^{k}$ such that $i \neq k ; i=1,2, \cdots, m$.

Step III: Form the matrix $d_{i j}^{k^{*}}=d_{i j}^{k}-e_{i}^{k}$ such that there exist at least one 0 in every row of $d_{i j}^{k^{*}}$.

Step IV: For each $\mathrm{k}=1,2 \cdots, \mathrm{m}$ compute $\mathrm{g}_{\mathrm{j}}^{\mathrm{k}}=\sum \mathrm{d}_{\mathrm{ij}}^{\mathrm{k}^{*}}$ for $\mathrm{i}=1,2, \cdots, \mathrm{m}$.

Step V: If $\mathrm{g}_{\mathrm{j}}^{\mathrm{k}}=0$ for $\mathrm{k}=\mathrm{q}$ and $\mathrm{j}=\mathrm{r}$ then the location $(\mathrm{q}, \mathrm{r})$ is an absolute point. 
Suppose if $\mathrm{g}_{\mathrm{j}}^{\mathrm{k}} \neq 0$ for any $\mathrm{j}$ and $\mathrm{k}$ then there is no absolute point at any location.

\section{Allocation algorithm based on absolute points}

Step 1: Form the Transportation cost matrix from the available information. The total number of units available at the sources may not be equal to the total number of units required at the destinations. The algorithm is suitable for both the balanced as well as the unbalanced transportation problem.

Step 2: Using the absolute point procedure given in section 4.1, identify the absolute point in the cost matrix, say, the location $(\mathrm{q}, \mathrm{r})$.

Step 3: Assign .

$$
\tilde{\mathrm{X}}_{\mathrm{qr}}=\left\{\begin{array}{l}
\tilde{\mathrm{a}}_{\mathrm{q}} \text { if } \min \left\{\mathfrak{R}\left(\tilde{\mathrm{a}}_{\mathrm{q}}\right), \mathfrak{R}\left(\tilde{\mathrm{b}}_{\mathrm{r}}\right)\right\}=\mathfrak{R}\left(\tilde{\mathrm{a}}_{\mathrm{q}}\right) \\
\tilde{\mathrm{b}}_{\mathrm{r}} \text { if } \min \left\{\mathfrak{R}\left(\tilde{\mathrm{a}}_{\mathrm{q}}\right), \mathfrak{R}\left(\tilde{\mathrm{b}}_{\mathrm{r}}\right)\right\}=\mathfrak{R}\left(\tilde{\mathrm{b}}_{\mathrm{r}}\right)
\end{array}\right.
$$

Step 4: The assignment will satisfy either the $\mathrm{q}^{\text {th }}$ row or $\mathrm{r}^{\text {th }}$ column and remove that row or column to form a reduced cost matrix.

Step 5: Adopting the absolute point procedure given in section 4.1 for the reduced cost matrix, identify the absolute point and repeat the Steps 2 to 4 until the distribution is complete.

\section{Illustration}

Example: Consider the Transportation problem with three sources S1, S2, S3 and three destinations D1, D2 and D3 with unit cost $C_{i j}$ of transporting the product from the source $\mathrm{Si}$ to destination $\mathrm{Dj}$ and the fuzzy supply $\tilde{\mathrm{a}}_{\mathrm{i}}$, the fuzzy demand $\tilde{b}_{j}$ of the product represented by symHFN and are given in Table 1. The objective is to find the fuzzy quantity of the product that should be transported from each source to each destination such that the total fuzzy cost of FTP is minimum. The problem considered is an unbalanced FTP as the total fuzzy supply is more than the total fuzzy demand. The proposed algorithm is applied to find the optimal solution without introducing the dummy source or destination.

\begin{tabular}{|c|c|c|c|c|}
\hline $\begin{array}{l}\text { Destination/ } \\
\text { Source }\end{array}$ & D1 & D2 & D3 & Fuzzy Supply \\
\hline S1 & 8 & 2 & 3 & $(\widetilde{20} ; 0.5,1)_{0.05}$ \\
\hline S2 & 2 & 12 & 8 & $(\widetilde{70} ; 0.5,1)_{0.05}$ \\
\hline S3 & 1 & 15 & 2 & $(\widetilde{30} ; 0.5,1) 0.05$ \\
\hline $\begin{array}{c}\text { Fuzzy } \\
\text { Demand }\end{array}$ & $(\widetilde{15} ; 0.5,1)_{0.05}$ & $(\widetilde{10} ; 0.5,1)_{0.05}$ & $(\widetilde{60} ; 0.5,1)_{0.05}$ & \\
\hline
\end{tabular}

Table 1.Cost matrix of the Example

Solution:

Step 1: The transportation cost matrix is given in Table 1.

Step 2: Adopting the absolute point procedure for the given cost matrix, Table 2 is obtained and it is observed that $\mathrm{g}_{2}^{1}=0$. Therefore the absolute point is identified at the location $(1,2)$.

Step 3: At the absolute point $(1,2)$, allocate the fuzzy quantity $\tilde{X}_{12}=\tilde{b}_{2}=(\widetilde{10} ; 0.5,1)_{0.05}$ because $\mathfrak{R}\left(\tilde{\mathrm{b}}_{2}\right)=3.06$ is less than $\mathfrak{R}\left(\tilde{\mathrm{a}}_{1}\right)=6.12$.

Step 4: Column 2 is satisfied and so drop the second column and update $\tilde{a}_{1}=(\widetilde{10} ; 0.5,1) 0.1$ 


\begin{tabular}{|c|c|c|c|c|c|c|c|}
\hline \multicolumn{3}{|c|}{$d_{i j}^{1}$} & \multirow{2}{*}{$\frac{\mathrm{e}_{\mathrm{i}}^{1} \text { (Row Minimum) }}{-}$} & \multicolumn{4}{|c|}{$\mathrm{d}_{\mathrm{ij}}^{1^{*}}$} \\
\hline- & - & - & & & - & - & - \\
\hline 6 & -10 & -5 & -10 & & 16 & 0 & 5 \\
\hline 7 & -3 & 1 & -13 & & 20 & 0 & 14 \\
\hline & & & & $\mathrm{g}_{\mathrm{j}}^{1}$ & 36 & 0 & 19 \\
\hline \multicolumn{8}{|c|}{ For Row 2} \\
\hline \multicolumn{3}{|c|}{$\mathrm{d}_{\mathrm{ij}}^{2}$} & $\mathrm{e}_{\mathrm{i}}^{2}$ (Row Minimum) & \multicolumn{4}{|c|}{$\mathrm{d}_{\mathrm{ij}}^{2^{*}}$} \\
\hline-6 & 10 & 5 & -6 & & 0 & 16 & 11 \\
\hline - & - & - & - & & - & - & - \\
\hline \multirow[t]{2}{*}{1} & -3 & 6 & -3 & & 4 & 0 & 9 \\
\hline & & & & $\mathrm{g}_{\mathrm{j}}^{2}$ & 4 & 16 & 20 \\
\hline \multicolumn{8}{|c|}{ For Row 3} \\
\hline \multicolumn{3}{|c|}{$d_{i j}^{3}$} & $\mathrm{e}_{\mathrm{i}}^{3}$ (Row Minimum) & \multicolumn{4}{|c|}{$\mathrm{d}_{\mathrm{ij}}^{3^{*}}$} \\
\hline-7 & 13 & -1 & -7 & & 0 & 2 & 6 \\
\hline-1 & 3 & -6 & -6 & & 5 & 9 & 0 \\
\hline- & - & - & - & & - & - & - \\
\hline & & & & $\mathrm{g}_{\mathrm{j}}^{3}$ & 5 & 11 & 6 \\
\hline
\end{tabular}

Table 2. Identifying AP for the cost matrix of the Example

Step 5: Drop the values in the second column of the matrices $\mathrm{d}_{\mathrm{ij}}^{\mathrm{k}}, \mathrm{d}_{\mathrm{ij}}^{\mathrm{k}}$ given in Table 2 and adopt the absolute point procedure for the reduced cost matrix. Table 3 is obtained and it is observed that $\mathrm{g}_{3}^{1}=0$ and $\mathrm{g}_{1}^{2}=0$. Therefore the absolute points are identified at two locations $(1,3)$ and $(2,1)$.

Step 6: At the absolute point $(1,3)$, allocate the fuzzy quantity $\tilde{\mathrm{X}}_{13}=\tilde{\mathrm{a}}_{1}=(\widetilde{10} ; 0.5,1)_{0.1}$ because $\mathfrak{R}\left(\tilde{\mathrm{a}}_{1}\right)=3.06$ is less than $\mathfrak{R}\left(\tilde{\mathrm{b}}_{3}\right)=18.36$. Row 1 is satisfied and update $\tilde{\mathrm{b}}_{3}=(\widetilde{50} ; 0.5,1)_{0.15}$

Step 7: At the absolute point $(2,1)$, allocate the fuzzy quantity $\tilde{X}_{21}=\tilde{b}_{1}=(\widetilde{15} ; 0.5,1)_{0.05}$ because $\mathfrak{R}\left(\tilde{b}_{1}\right)=4.59$ is less than $\mathfrak{R}\left(\tilde{\mathrm{a}}_{2}\right)=21.42$. Column 1 is satisfied and update $\tilde{\mathrm{a}}_{2}=(\widetilde{55} ; 0.5,1) 0.1$

Step 8: After dropping the first row and first column, the reduced cost matrix is left with the cells $(2,3)$ and $(3,3)$. At the location $(3,3)$, allocate the fuzzy quantity $\tilde{\mathrm{X}}_{33}=\tilde{\mathrm{a}}_{3}=(\widetilde{30} ; 0.5,1)_{0.05}$ because $\mathfrak{R}\left(\tilde{\mathrm{a}}_{3}\right)=9.18$ is less than $\mathfrak{R}\left(\tilde{\mathrm{b}}_{3}\right)=15.3$. Row 3 is satisfied and update $\tilde{\mathrm{b}}_{3}=(\widetilde{20} ; 0.5,1) 0.2$

Step 9: At the location (2,3), allocate the fuzzy quantity $\tilde{X}_{23}=\tilde{b}_{3}=(\widetilde{20} ; 0.5,1)_{0.2}$ because $\mathfrak{R}\left(\tilde{b}_{3}\right)=6.12$ is less than $\mathfrak{R}\left(\tilde{\mathrm{a}}_{2}\right)=16.83$. Column 3 is satisfied and update $\tilde{\mathrm{a}}_{2}=(\widetilde{35} ; 0.5,1)_{0.3}$

Step 10: The excess supply $\tilde{\mathrm{a}}_{2}=(\widetilde{35} ; 0.5,1) 0.3$ is left at the source $\mathrm{S} 2$ and it indicates the management that a suitable decision has to be taken to increase demand at certain destinations.

Thus without introducing dummy destination, the solution of the unbalanced FTP is obtained as $\tilde{\mathrm{X}}_{12}=(\widetilde{10} ; 0.5,1)_{0.05}$, $\tilde{\mathrm{X}}_{13}=(\widetilde{10} ; 0.5,1)_{0.1}, \tilde{\mathrm{X}}_{21}=(\widetilde{15} ; 0.5,1)_{0.05}, \tilde{\mathrm{X}}_{23}=(\widetilde{20} ; 0.5,1)_{0.2}, \tilde{\mathrm{X}}_{33}=(\widetilde{30} ; 0.5,1)_{0.05}$ with total transportation cost of $(\widetilde{300} ; 0.5,1) 2.2$ equivalent to the crisp cost 91.8 . 


\begin{tabular}{|c|c|c|c|c|c|c|c|}
\hline \multicolumn{3}{|c|}{$d_{i j}^{1}$} & $\mathrm{e}_{\mathrm{i}}^{1}$ (Row Minimum) & & \multicolumn{3}{|c|}{$\mathrm{d}_{\mathrm{ij}}^{1^{*}}$} \\
\hline- & - & - & - & & - & - & - \\
\hline 6 & - & -5 & -5 & & 11 & - & 0 \\
\hline 7 & - & 1 & 1 & & 6 & - & 0 \\
\hline & & & & $\mathrm{g}_{\mathrm{j}}^{1}$ & 17 & - & 0 \\
\hline \multicolumn{8}{|c|}{ For Row 2} \\
\hline \multicolumn{3}{|c|}{$\mathrm{d}_{\mathrm{ij}}^{2}$} & $\mathrm{e}_{\mathrm{i}}^{2}$ (Row Minimum) & \multicolumn{4}{|c|}{$\mathrm{d}_{\mathrm{ij}}^{2 *}$} \\
\hline-6 & - & 5 & -6 & & 0 & - & 11 \\
\hline- & - & - & - & & - & - & - \\
\hline 1 & - & 6 & 1 & & 0 & - & 5 \\
\hline & & & & $\mathrm{g}_{\mathrm{j}}^{2}$ & 0 & - & 16 \\
\hline \multicolumn{8}{|c|}{ For Row 3} \\
\hline & $d_{i j}^{3}$ & & $\mathrm{e}_{\mathrm{i}}^{3}$ (Row Minimum) & \multicolumn{4}{|c|}{$\mathrm{d}_{\mathrm{ij}}^{3 *}$} \\
\hline-7 & - & -1 & -7 & & 0 & - & 6 \\
\hline-1 & - & -6 & -6 & & 5 & - & 0 \\
\hline \multirow[t]{2}{*}{ - } & - & - & - & & - & - & - \\
\hline & & & & $\mathrm{g}_{\mathrm{j}}^{3}$ & 5 & - & 6 \\
\hline
\end{tabular}

\section{Advantages of the proposed algorithm}

The proposed algorithm based on the absolute points reduces the dimension of a FTP so that the computation process takes less time.

The identification of APs at any iteration is independent of the supply and demand constraints and depends on the values of $\mathrm{C}_{\mathrm{ij}}$ only. Hence the proposed algorithm is more suitable for unbalanced FTP.

Identification of the absolute points in the Transportation table helps to manage dynamic conditions which affect the cost coefficients, such as a strong national currency or foreseeable future traffic congestion.

\section{Conclusion}

An attempt is made to develop a new algorithm based on the absolute points to solve the unbalanced FTP without introducing dummy source or destination. The absolute point is a location that must be loaded in any optimal solution of FTP and so the solution obtained by the proposed algorithm is optimal. The excess supply or demand that is not loaded in the optimal solution indicates the management that it has to take a suitable decision such as increasing a ware-house stocking level or advertising efforts to increase demand at certain markets. So the proposed algorithm provides the added information of transporting the excess availability from dummy supply point to appropriate destination to meet future demands to a variety of distribution problems.

\section{Conflicts of Interest}

The author declares no conflict of interest.

\section{References}

1. Adlakha V, Kowalski K. A quick sufficient solution to the more-for-less paradox in the transportation problem. International Journal of Management Science 1998; 26(4):541-547.

2. Arsham H, Khan AB. A simplex-type algorithm for general transportation problems: an alternative to stepping stone. j. oper. res. Soc 1989; 40: 581-590.

3. Basirzadeh H. An approach for solving fuzzy transportation problem. Applied Mathematical Science 2011; 5: $1549-1566$. 
4. Das MK, Baruah HK. Solution of the transportation problem in fuzzified form journal of fuzzy mathematics 2007; 15: 79-95.

5. Rani D, Gulati TR, Amit KA. A method for unbalanced transportation problems in fuzzy environment2014; 39(3): 573-581.

6. Gani AN, SamuelAE, Anuradha D. Simplex type algorithm for solving fuzzy transportation problem. Tamsui Oxf. J. Math. Sci 2011; 27: 89-98.

7. Hitchcock FL. The distribution of a product from several resources to numerous localities, journal of mathematical physics 1941; 20: 224-230.

8. Rathi K, Balamohan S. A mathematical model for subjective evaluation of alternatives in fuzzy multi-criteria group decision making using copras method. international journal of fuzzy systems 2016; 19. 10.1007/s40815-016-0256-z.

9. RathiK, BalamohanS. Representation and ranking of fuzzy numbers with heptagonal membership function using value and ambiguity index, applied mathematical sciences2014; 87(8): 4309-4321.

10. Saad OM, Abbas SA. A parametric study on transportation problem under fuzzy environment. journal of fuzzy mathematics 2003; 11: 115-124.

11. Zadeh LA. Fuzzy sets. inf. control 1965; 8: 338-353.

12. Adlakha V, Kowalski K. A quick sufficient solution to the more-for-less paradox in the transportation problem. International Journal of Management Science 1998; 26(4):541-547.

13. Arsham H, Khan AB. A simplex-type algorithm for general transportation problems: an alternative to stepping stone. j. oper. res. Soc 1989; 40: 581-590.

14. Basirzadeh H. An approach for solving fuzzy transportation problem. Applied Mathematical Science 2011; 5: 1549-1566.

15. Das MK, Baruah HK. Solution of the transportation problem in fuzzified form journal of fuzzy mathematics 2007 ; 15: 79-95.

16. Rani D, Gulati TR, Amit KA. A method for unbalanced transportation problems in fuzzy environment2014; 39(3): 573-581.

17. Gani AN, SamuelAE, Anuradha D. Simplex type algorithm for solving fuzzy transportation problem. Tamsui Oxf. J. Math. Sci 2011; 27: 89-98.

18. Hitchcock FL. The distribution of a product from several resources to numerous localities, journal of mathematical physics 1941; 20: 224-230.

19. Rathi K, Balamohan S. A mathematical model for subjective evaluation of alternatives in fuzzy multi-criteria group decision making using copras method. international journal of fuzzy systems $2016 ; 19$. 10.1007/s40815-016-0256-z.

20. RathiK, BalamohanS. Representation and ranking of fuzzy numbers with heptagonal membership function using value and ambiguity index, applied mathematical sciences2014; 87(8): 4309-4321.

21. Saad OM, Abbas SA. A parametric study on transportation problem under fuzzy environment. journal of fuzzy mathematics 2003; 11: 115-124.

22. Zadeh LA. Fuzzy sets. inf. control 1965; 8: 338-353. 